\section{Late hematogenous seeding and infection of a femoropopliteal Omniflow II bypass}

\author{
Hozan Mufty $\mathbb{D}$, Sabrina Houthoofd, Kim Daenens and \\ Inge Fourneau
}

\begin{abstract}
Introduction: Omniflow II is promoted as an infection-resistant vascular graft. It is used to treat vascular graft infection; nevertheless, early graft infection has been reported.

Report: A 7I-year-old patient was treated with an Omniflow II bypass for a non-healing diabetic foot ulcer. Seven months postoperatively, late infection occurred secondary to hematogenous spread from a persistent foot infection.

Conclusion: We report on the first case of late infection of an Omniflow II vascular graft caused by hematogenous spread. Despite promising results of the Omniflow II graft in the treatment of vascular graft infection, late infection may not be avoided.
\end{abstract}

\title{
Keywords
}

Omniflow II, infection, vascular graft

\section{What this paper adds}

Omniflow II is not infection resistant and hematogenous infection is possible, even in a late phase.

\section{Introduction}

Omniflow II (Lemaitre Vascular, Burlington, MA, USA) is a biosynthetic composite of a polyester mesh endoskeleton with cross-linked ovine collagen to encourage rapid incorporation. The graft is promoted to be infection resistant. However, reports dealing with the role of Omniflow II in the treatment of vascular graft infection (VGI) are limited to case series. ${ }^{1,2}$ The role of Omniflow II in the prevention of VGI is not well studied. ${ }^{3,4}$ Early infection ( $<30$ days) of Omniflow II has been reported. ${ }^{3,5}$ Until now, no reports of late infection are reported.

We report a patient with late infection of an Omniflow II bypass secondary to hematogenous spread of infection from a diabetic foot ulcer.

\section{Report}

A 71-year-old male patient was admitted to the emergency department with an infectious syndrome $\left(38.3^{\circ} \mathrm{C}\right.$, white blood cell: $13.48 \times 10^{9} / \mathrm{L}, \mathrm{CRP}: 286.7 \mathrm{mg} / \mathrm{L}$ ).
The patient had a history of iliofemoral bypass grafting (external iliac artery to deep femoral artery), aortic valve bioprosthesis and total hip replacement (all $>7$ years ago). He was treated 11 months earlier with a femoropopliteal bypass above the knee using an Intergard Synergy vascular graft (Maquet, Getinge group, NJ USA) and an intertarsal forefoot amputation on the left side for a non-healing diabetic foot ulcer.

As, three months later, an occlusion of the femoropopliteal bypass occurred with subsequent dehiscence and secondary infection of the amputation wound, a redo femoropopliteal bypass (End-to-End anastomosis on P2 segment) was created using an Omniflow II graft. A new graft tunnel was created. The proximal anastomosis was sutured on the side-to-end on the iliofemoral bypass. The occluded femoropopliteal artery was not well incorporated due to seroma formation. This

Department of Vascular Surgery, University Hospitals Leuven, Leuven, Belgium

\section{Corresponding author:}

Hozan Mufty, Department of Vascular Surgery, University Hospitals Leuven, Herestraat 49, Leuven 3000, Belgium.

Email: Hozan.mufty@uzleuven.be 
bypass was removed in toto. Cultures taken here did not reveal bacterial growth. A wound debridement with Chopart's amputation was performed without closure of the wound. Broad spectrum antibiotics were started empirically.

Based on wound cultures (methicillin sensitive Staphylococcus aureus (MSSA), Candida albicans, Klebsiella pneumonia), flucloxacillin, meropenem and fluconazole were administered for four weeks and levofloxacin in monotherapy thereafter. Levofloxacin was stopped sixweeks after secondary closure of the wound. However, four months after complete healing of the foot wound, a new dehiscence occurred. At the time of presentation at the emergency department with the infectious syndrome, the bypass was still patent with pulsations at the level of the foot.

Blood cultures were positive for MSSA. Magnetic resonance imaging of the foot could not demonstrate osteomyelitis. A transesophageal echocardiography showed abscess formation at the level of the aortic root with vegetations on the prosthesis valve leaflets.

Adjuvant positron-emission tomography/computed tomography (PET/CT) confirmed endocarditis and suspected infection of the Omniflow II bypass and septic emboli in both lungs. Persistent infection at the level of the calcaneus was also observed. (Figure 1) The patient underwent urgent cardiac surgery. Perioperatively, the Omniflow II bypass occluded. Once the patient was stabilized post-cardiac surgery, limb salvage was not possible anymore. Subsequently, an above the knee amputation was inevitable. During this procedure, massive pus around the Omniflow II bypass was noticed. The consistency of the graft was jelly like, suggestive for degeneration of the collagen covering. The iliofemoral bypass was well incorporated over the whole length. Cultures from both the cardiac valve and bypass were negative. Adjuvant PCR analysis confirmed $S$. aureus as the causative pathogen.

\section{Discussion}

Hematogenous infection of a vascular graft is the second most common cause of VGI, after intraoperative bacterial contamination. The risk of hematogenous seeding is particularly high before the pseudo-intimal formation on the graft is completed, i.e. $<2$ months. ${ }^{6,7}$ This early infection of Omniflow II has been reported. ${ }^{3,5}$ Few studies focusing on the longterm outcome of Omniflow II graft are published. They report early graft infection rates ranging from 0 to $1.9 \%$. No late graft infections are reported. ${ }^{3,4,8,9}$ Neufang et al. reported 4 out of 205 bypasses $(1.9 \%)$ to be involved in deep wound infection or infected hematoma. In all cases, the Omniflow II was exposed due to wound dehiscence, but could be preserved. ${ }^{3}$ In
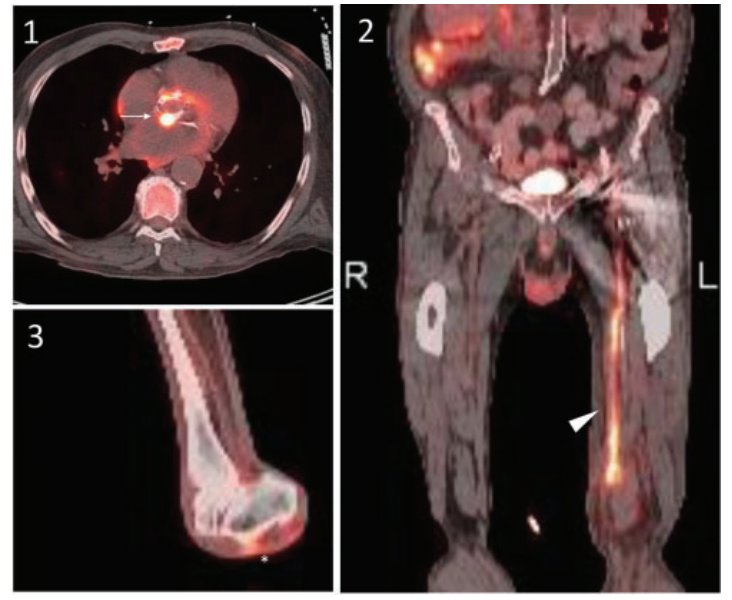

Figure I. Positron-emission tomography/computed tomography showing hypermetabolism of the aortic bioprosthesis (image I, arrow, axial view), of the Omniflow II bypass (image 2, arrow head, coronal view) and of the calcaneus (image 3, asterix, sagittal view), suggestive for endocarditis, infection of the bypass and osteomyelitis, respectively.

our case, the bypass was implanted in a patient with a non-healing diabetic foot ulcer without evidence of surgical site infection postoperatively. The infection occurred four months after complete healing of the foot ulcer and seven months after creation of the bypass. The concomitant infectious foci in lung and pericarditis were suggestive for hematogenous spread. This case proves that an Omniflow II bypass cannot be considered $100 \%$ resistant to infection. Nevertheless, Omniflow II used in the treatment of VGI has encouraging results with no reinfection reported up to 66 months. ${ }^{1,2,10}$

\section{Conclusion}

We report on a case of late infection of an Omniflow II vascular graft caused by hematogenous spread. Despite promising results of the Omniflow II graft in the treatment of VGI, late infection may not be avoided.

\section{Declaration of conflicting interests}

The author(s) declared no potential conflicts of interest with respect to the research, authorship, and/or publication of this article.

\section{Funding}

The author(s) received no financial support for the research, authorship, and/or publication of this article.

\section{Informed consent}

Informed consent has been obtained for publication of the case report and possible accompanying images. 


\section{ORCID iD}

Hozan Mufty (D) https://orcid.org/0000-0003-4751-8862

\section{References}

1. Betz T, Neuwerth D, Steinbauer M, et al. Biosynthetic vascular graft: a valuable alternative to traditional replacement materials for treatment of prosthetic aortic graft infection? Scand J Surg 2019; 108: 291-296.

2. Wiltberger G, Matia I, Schmelzle M, et al. Mid- and long-term results after replacement of infected peripheral vascular prosthetic grafts with biosynthetic collagen prosthesis. J Cardiovasc Surg 2014; 55: 693-698.

3. Neufang A, Duenschede F, Espinola-Klein C, et al. Contemporary results with the biosynthetic glutaraldehyde denatured ovine collagen graft (Omniflow II) in femoropopliteal position. J Vasc Surg 2020; 71: 1630-1643.

4. Dunschede F, Youssef M, Stabrauskaite J, et al. Omniflow-II(TM) for critical limb ischemia: long-term results. Chirurg 2017; 88: 233-238.
5. Neufang A, Dorweiler B, Espinola-Klein C, et al. Outcomes of complex femorodistal sequential autologous vein and biologic prosthesis composite bypass grafts. $J$ Vasc Surg 2014; 60: 1543-1553.

6. Wilson WR, Bower TC, Creager MA, et al. Vascular graft infections, mycotic aneurysms, and endovascular infections: a scientific statement from the American Heart Association. Circulation 2016; 134: e412-e460.

7. Chaudhary R and Simmons RL. Pathogenesis of vascular graft infections. J Vasc Surg 1991; 13: 755-756.

8. Dunschede F, Stabrauskaite J, Weisser G, et al. Crural bypass for critical lower limb ischemia with omniflow II prosthesis. Thorac Cardiovasc Surg 2016; 64: 311-315.

9. Koch G, Gutschi S, Pascher O, et al. Analysis of 274 omniflow vascular prostheses implanted over an eightyear period. Aust N Z J Surg 1997; 67: 637-639.

10. Topel I, Stigler T, Ayx I, et al. Biosynthetic grafts to replace infected prosthetic vascular bypasses: a singlecenter experience. Surg Infect 2017; 18: 202-205. 